\title{
PENSAMIENTO, SABER Y RACIONALIDAD AMBIENTAL
}

Oswaldo Báez. Tobar

Universidad Central del Ecuador

Recibido: 12 - octubre - 2015, aprobado 24 - noviembre - 2015

\section{Resumen}

La construcción del pensamiento ambiental y de una filosofía que incorpore la dimensión ambiental, están presentes en las reflexiones académicas contemporáneas. El pensamiento ambiental está en proceso de construcción teórica, cruza de manera transversal y compleja la totalidad de las disciplinas con el fin de estructurar conceptos y valores que ayuden a la humanidad actual a redefinir su relación con la naturaleza y a pasar de una actitud de dominio a una actitud de respeto hacia ella. El presente estudio es una aproximación al pensamiento ambiental desde diversos niveles y enfoques con el propósito de alcanzar una visión integral del ambiente. Aborda aspectos fundamentales de ecología, sistemas ecológicos y la biósfera, analiza el pensamiento ambiental en toda su amplitud y complejidad, para culminar con el saber y la racionalidad ambiental, cuyo potencial de transformación social apenas se empieza a vislumbrar. El nuevo pensamiento ambiental resultante de la síntesis de las ciencias naturales y las ciencias sociales modernas, la filosofía y la axiología, está ganando un importante espacio en las reflexiones académicas de la presente década y se espera que ilumine el camino de la humanidad en este siglo. Finalmente, el estudio presenta una breve revisión del pensamiento ambiental latinoamericano que se viene configurando con especial interés en años recientes.

Palabras clave: ecología, ecosistema, medio ambiente, pensamiento ambiental, epistemología ambiental, racionalidad ambiental.

\begin{abstract}
The construction of environmental thought and a philosophy that incorporates the environmental dimension are present in contemporary academic reflections. Environmental thinking is in theoretical construction process, that transversely and complexly crosses all the disciplines in order to structure concepts and values that help present humanity to redefine its relationship with nature and thus, to pass from a domain attitude to an attitude of respect towards her. The present study is an approach to environmental thinking from different levels and points of view, in order to achieve a holistic view of the environment. It addresses key aspects of ecology, ecological systems and the biosphere, analyzes environmental thinking in all its breadth and complexity, culminating with environmental knowledge and rationality, which potential for social transformation is just beginning to glimpse. The new environmental thinking resulting from the synthesis between the modern natural sciences and social sciences, philosophy and axiology, is gaining an important place in academic reflections of the present decade and is expected to enlighten the path of humanity in this century. Finally, the study presents a brief review of Latin American environmental thinking which has been setting, with special interest in recent years.
\end{abstract} Keywords: ecology, ecosystem, environment, environmental thinking, environmental epistemology, environmental soundness. 


\section{Contribuciones de la ecología}

La ecología es una ciencia entre vieja y nueva. Si se considera el enfoque ecológico de la relación hombre-naturaza es muy antigua, pues están presentes algunos elementos de carácter "protoecológico" en varias obras de filósofos y poetas como Lucrecio, Virgilio y Teofrasto, como en otros pensadores de la antigüedad, en especial en las civilizaciones de oriente así como en algunas religiones. La ecología como ciencia dotada de un cuerpo de conocimientos definidos y una metodología establecida es una ciencia reciente, rica en cambios y de historia agitada (Francesco di Castri, 1981).

La palabra ecología viene de la raíz griega oikos que significa casa o lugar donde se vive. Como disciplina científica la ecología es la ciencia que estudia las relaciones entre un organismo y su entorno. Desde el nacimiento de esta ciencia se entendió por ecología al conjunto de conocimientos referentes a la economía de la naturaleza. La ecología es el estudio de las interrelaciones entre los seres vivos y su ambiente físico y biótico.

Para Charles Elton la ecología es la historia natural científica y se encarga de la sociología y economía de los animales. Según Clemens es la ciencia de la comunidad y para Eugene Odum la ecología es el estudio de la estructura y función de la naturaleza. Ver Kormondy (1973). La ecología en sentido estricto es la biología de los ecosistemas; describe la naturaleza en términos de materia, energía y organización. El número de especies y su diversidad son aproximaciones a una medida de organización (Margalef, 1980, 1983). En una visión moderna la ecología es la ciencia natural que estudia las relaciones sistémicas entre los individuos, dentro de ellos y entre ellos y el medio ambiente (definición funcional). Es el estudio científico de la distribución y abundancia de los organismos que interactúan con su medio ambiente en un tiempo y espacio definidos (definición estructural). Es la ciencia del medio ambiente (definición holística). Sarmiento (2001).

La evolución histórica de la ecología revela a esta disciplina como una de las más ricas y apasionantes en la historia de las ciencias. En 1870 se tomó conciencia de que existe una verdadera ciencia ecológica después de la formulación conceptual hecha por Haeckel. Empero, la ecología tiene un desenvolvimiento distinto al de las otras ciencias naturales: es la ciencia de síntesis. Según Francesco di Castri se trata del gran diálogo dialéctico entre dos tipos de ciencias, por un lado las ciencias del análisis con claro enfoque reduccionista que disocian cada vez más las estructuras para estudiarlas en profundidad y por otro las ciencias de síntesis u holísticas cuyo mayor ejemplo es la ecología, que se esfuerza por comprender el sistema en su conjunto mediante las interrelaciones entre todos sus elementos.

La ecología, en verdad, se mueve entre las dos tendencias científicas, ambas son importantes y complementarias para el conocimiento de la naturaleza. La ciencia ecológica como toda ciencia tiende también al análisis, sin embargo predomina en la ecología el enfoque holístico de conjuntos más o menos amplios como son las poblaciones, comunidades y ecosistemas.

Se reconoce que el tratamiento analítico en las ciencias biológicas alcanzó gran supremacía con la bioquímica, biofísica, biología celular y molecular y genética 
molecular, disciplinas que alcanzaron notable prestigio académico y alto desarrollo, a la vez que influyeron en el control de la política científica y constituyeron el soporte de la biotecnología moderna.

En ese contexto general de las ciencias se abre paso la ecología con un gran valor heurístico y ético. Ha dado testimonio de su razón de ser en el conjunto de las ciencias como en la sociedad contemporánea, al punto que se ha convertido en una de las ciencias biológicas de mayor desarrollo y difusión en todos los estratos sociales y niveles de educación y formación profesional. Como ciencia moderna y compleja busca nuevos caminos y nuevas relaciones que se expresan en el conocimiento de la naturaleza y del hombre dentro de ella.

En la evolución de la ciencia ecológica se identifica varias etapas; estas corresponden a distintos períodos desde el punto de vista cronológico y conceptual. En los siglos XVIII y XIX predominaron los estudios descriptivos de la naturaleza; fue la época de exploradores y naturalistas y sus fascinantes relatos de historia natural, seguida de la descripción de las especies vegetales y animales en su entorno natural.

Tales descripciones fueron adquiriendo poco a poco mayor rigor científico para dar origen a la autoecología que es la ecología de una sola especie. Sin embargo, el estudio de la especie y sus interrelaciones con el ambiente es solo una parte de la ecología. Surgió más adelante la ecología de poblaciones y la ecología de las comunidades o sinecología, para estudiar el conjunto de especies de una unidad natural determinada, sus interrelaciones y su dinamia; en esta fase surgieron los conceptos de comunidad biológica, cadena alimenticia, pirámide energética, dinámica de las poblaciones, entre otros. El nuevo y atractivo enfoque sinecológico fue bastante fructífero y permitió enriquecer el conocimiento de la naturaleza; sin embargo, pronto se hizo evidente la falta de una unidad de estudio de la ecología. Surgió entonces la necesidad de identificar la unidad de constitución y funcionamiento de la naturaleza: el sistema ecológico o ecosistema.

\section{Conceptualización del ecosistema}

El término ecosistema fue propuesto en 1936 por Artur George Tansley quien lo usó para referirse a todo el sistema físico incluyendo el complejo de organismos a los que no se puede desligar de su ambiente espacial con el que forman un solo sistema. Empero, fue Raymond Lindeman, en 1942, quien al estudiar la cadena alimenticia e incorporar el flujo de la energía y el ciclo de la materia, formuló la teoría de la estructura y funcionamiento del ecosistema con lo cual estableció las bases conceptuales y metodológicas para su conocimiento integral.

Así surgió la concepción teórica del ecosistema como la unidad natural constituida por los componentes vivientes: plantas, animales, hongos y microorganismos, y los componentes no vivientes o abióticos como el suelo y sus minerales, el agua, la atmósfera y los factores del clima, todo lo cual integra una unidad funcional e interdependiente. Según Toledo (1987) el ecosistema -última unidad a la que puede reducirse todo paisaje natural- constituye una entidad dotada de una arquitectura, organización y funcionamiento determinado. Y la ecología a 
través del ecosistema encuentra la unidad en la compleja diversidad del paisaje, descubre la estructura funcional de la naturaleza, en la cual los flujos de la energía y ciclos de la materia están en equilibrio dinámico. Ello implica la capacidad de los ecosistemas de automantenerse y autoreproducirse según las propias leyes de la naturaleza. Es decir, revela la constitución y el funcionamiento de la 'maquinaria' mediante la cual se renueva la biosfera.

El ecosistema según Odum (1969) es la unidad funcional básica porque incluye tanto organismos como ambiente climático, cada uno de los cuales influye en las propiedades del otro, siendo necesarios ambos para la preservación de la vida como la tenemos en la Tierra. Para Ramón Margalef (1980) ecosistema es el sistema formado por individuos de muchas especies en el seno de un ambiente de características definidas e implicadas en procesos dinámicos e incesantes de interacción, ajuste y regulación... un retazo cualquiera de la biosfera es un ecosistema. La palabra no se usa en el sentido de unidad concreta, sino en el sentido de organización.

Así, la concepción teórica de ecosistema pasó a ser el constructo intelectual más adecuado para entender la naturaleza y para delimitar la unidad y el objeto de estudio de la ecología. Tal concepto se consolidó al articularse con la Teoría General de Sistemas de Ludwing von Bertalanffy que en esencia postula la interrelación entre los elementos del todo. Con este enfoque avanzó el conocimiento de la estructura y fisiología de ecosistemas, que hizo posible la formulación de modelos de sistemas ecológicos.

Cabe destacar que el mayor desarrollo de las ciencias ecológicas coincidió con la época de crecimiento económico que llevó a la crisis medio ambiental de los años 70. Eso generó una nueva conciencia sobre lo limitado de los recursos naturales, el peligro de su agotamiento, la crisis energética, la contaminación a escala mundial, la creciente desigualdad entre los países industrializados y los del Tercer Mundo.

En ese contexto y bajo el concepto de "Una sola tierra" la Organización de las Naciones Unidas organizó la Conferencia Mundial sobre el Medio Humano, en Estocolmo en el año 1972. Por primera vez se debatió en el foro internacional más importante el problema de la destrucción del medio ambiente. A partir de 1972 la cuestión ecológica ocupó la atención de la academia y del gran público. La ecología pasó a ocupar desde entonces la atención mundial. Frente a la potencial crisis ecológica los políticos y planificadores empezaron a buscar en la ecología la respuesta a las nuevas preguntas; pero, la ciencia ecológica no se hallaba todavía en capacidad de dar respuestas debido a que no se había incluido en el análisis los factores temporales, sociales y económicos. Cuando se incorporó en el análisis estos factores se produjo una gran conmoción en todos los ámbitos del saber, lo cual tuvo implicaciones en las ciencias naturales y en las ciencias sociales, así como en la conciencia individual y colectiva.

En los países industrializados como Estados Unidos y los países de Europa, así como en el Tercer Mundo surgieron grupos y movimientos sociales preocupados por el medio ambiente. Nació el ecologismo con sus múltiples vertientes, desde 
las moderadas hasta las más radicales, para cuestionar a la civilización tecnológica por ser la responsable, directa o indirecta, de la situación del medio ambiente. El ecologismo asimiló los conceptos de la ecología y los usó como bandera de lucha por la defensa de la naturaleza.

En espacios académicos los ecólogos de varios países prendieron la voz de alarma y encabezaron importantes movimientos sociales por el cambio de la sociedad, lograron atraer la atención de los gobiernos respecto de los problemas del medio ambiente así como sobre las cuestiones ecológicas del desarrollo. Con las evidencias que proporcionó la ciencia ecológica, economistas y planificadores formularon una propuesta novedosa: el ecodesarrollo, que encontró en América Latina un espacio propicio para su promoción. Proliferaron iniciativas académicas e investigativas dirigidas a diagnosticar los problemas ambientales de los países y a generar propuestas para el desarrollo ajustadas a las condiciones ecosistémicas y socioambientales, con lo cual se configuró el discurso del ecodesarrollo. El concepto de ecodesarrollo tuvo una connotación regional, pues fue entendido como el desarrollo posible dentro de las circunstancia de cada región, con tecnologías alternativas y participación de los pueblos; en oposición al desarrollo unidimensional del capitalismo moderno. En otro ámbito la ecología constituyó el soporte científico de la conservación de la naturaleza; surgió así la ecología de la conservación.

\section{La biosfera en la discusión mundial}

El concepto de biosfera propuesto por Verdnadsky en 1926 se lo acepta en el ámbito científico. La biosfera es la "parte de tierra donde existe vida, pero es además representativa del nivel superior de organización de la materia en la Tierra". (Hutchinson, 1972).

El ámbito de atención de la comunidad internacional sobrepasó los límites de los ecosistemas y aun de las regiones biogeográficas para englobar a toda la biosfera. El futuro de la biosfera y la responsabilidad del hombre fue la principal preocupación de las ciencias en las últimas décadas del siglo XX como lo es ahora en las primeras décadas del siglo XXI. La comunidad científica internacional así como la comunidad de naciones representadas por la ONU iniciaron programas de gran aliento orientados al estudio del medio ambiente y de la biosfera; ejemplo de ello constituyeron el Programa de las Naciones Unidas para el Medio Ambiente PNUMA y el Programa del Hombre y la Biosfera MAB de la UNESCO (por las siglas en inglés).

Hasta antes del Programa el Hombre y la Biosfera, MAB en 1977 se abordó los efectos de la actividad humana en la biosfera con un enfoque externalista, es decir, desde "afuera". Más tarde se tomó en cuenta al hombre como integrante de los sistemas ecológicos y de la biosfera, lo que implica el estudio del hombre dentro de la biosfera, pues revela el proceso evolutivo y reproduce en la ciencia la evolución de la naturaleza y la humanidad.

Este enfoque significó una revolución conceptual y metodológica que tuvo incidencia en el ámbito científico, en la conservación de la naturaleza y el medio 
ambiente. Así, después de varios años perdidos para el desarrollo social y para el ambiente, por efecto de la dependencia económica, la sobreexplotación de los recursos naturales y la contaminación ambiental creciente, la ONU convocó a la Conferencia de las Naciones Unidas sobre el Medio Ambiente y Desarrollo CNUMAD, la Conferencia Cumbre de la Tierra, en Río de Janeiro en junio de 1992. La Conferencia fue uno de los acontecimientos de mayor trascendencia en las últimas décadas del siglo XX, pues, llevó el tema ambiental a la agenda geopolítica mundial y abrió el debate sobre los problemas del medio ambiente como subproducto del modelo de desarrollo tecnológico industrial. La Conferencia llegó a los siguientes acuerdos: Convención Marco sobre Cambio Climático; Convención sobre Diversidad Biológica; Declaración sobre Medio Ambiente y Desarrollo y la Agenda 21.

Científicos e intelectuales de América Latina con apoyo del PNUD y del BID prepararon el documento: Nuestra Propia Agenda, con la posición y planteamientos de los países latinoamericanos. En este documento se encuentra la simiente del pensamiento ambiental latinoamericano. Roberto Guimaráes expresó: “Desde la perspectiva latinoamericana y tercermundista la Cumbre de la Tierra significó el reconocimiento de que la crisis actual representa el agotamiento de un estilo de desarrollo ecológicamente depredador, socialmente perverso y políticamente injusto", (Guimaráes, 1992).

La trascendencia de la Conferencia de Río fue importante en el ámbito del pensamiento ambiental y en la política ambiental nacional e internacional al socializar la reflexión sobre la precariedad de los sistemas naturales para mantener la vida en el planeta, la inviabilidad del crecimiento económico ilimitado, la finitud de los recursos naturales que sustentan la vida humana, la incidencia planetaria de los cambios climáticos, la disminución de la diversidad biológica, la degradación de los suelos y la desertificación.

De la Conferencia de Estocolmo a la Cumbre de Río los conceptos y las corrientes de pensamiento avanzaron sustancialmente: desde los tiempos en que se postuló la tesis de "Una sola Tierra", se pretendía hallar la solución a todos los problemas ambientales en la ciencia y la tecnología, hasta el surgimiento de la tesis del desarrollo sostenible entendido como el desarrollo que satisface las necesidades de la generación actual sin comprometer la posibilidad de las futuras generaciones de satisfacer sus propias necesidades.

El concepto de desarrollo sostenible o desarrollo sustentable -que tuviera amplia difusión desde los organismos internacionales y entidades de planificación de muchos países-, recibió duras críticas por ser eufemista, ambiguo e indefinible, porque lleva implícito el crecimiento ilimitado, implica desarrollo dentro de los mismos sistemas de producción y consumo de los países industrializados y deja abierto el camino para el crecimiento de los países desarrollados como por los países del Tercer Mundo que buscaron imitar el desarrollo de los primeros, lo cual es imposible por lo limitado de los recursos naturales y la incapacidad de los sistemas ecológicos para absorber los desechos. Por todo esto, el desarrollo sostenible es una utopía. Los recursos de la naturaleza son finitos, la Tierra como sumidero 
de desechos ha superado la capacidad de absorción; pues, una vez que se sobrepasa su capacidad de resiliencia ambiental el proceso es irreversible.

En el año 2002 se reunió la Cumbre Mundial sobre Desarrollo Sostenible CMDS en Johannesburgo con el propósito de evaluar la aplicación de las recomendaciones y promesas de la Conferencia de Río-92 y planificar las acciones futuras. La CMDS produjo varios resultados: la Declaración Política; el Plan para promover la Agenda 21, los Diálogos de las Partes sobre el agua, energía, salud, agricultura y biodiversidad (WEHAB por las siglas en inglés). En la conferencia de Naciones Unidas del año 2012 denominada Río+20 se aprobó el documento "El futuro que queremos". Contiene los lineamientos que cada país debería seguir para lograr el desarrollo sostenible por medio de la llamada "economía verde". El documento de Río +20 fue calificado como un texto vacío de compromisos, ante la mayoría de participantes, a la vez que puso en evidencia las diferencias insalvables entre los 193 Estados representados. La "economía verde" sería la nueva panacea para solucionar los problemas ambientales a nivel planetario, pero ha recibido muchas críticas de sectores académicos y ambientalistas, pues se interpreta como un conjunto de medidas cosméticas para apuntalar el sistema económico-financiero internacional, sin enfrentar los problemas ambientales.

En la evaluación de los resultados de Río+20 hubo una gama de percepciones contrapuestas: entre el éxito y el fracaso, entre ilusiones y desilusiones, dado que la conferencia se realizó en un período conflictivo en el que la humanidad se enfrentaba a un escenario geopolítico caracterizado por una mayor polarización entre los países ricos y los países pobres y dependientes, y cuando la crisis ecológica y económica alcanzaba dimensiones mundiales, pero faltó la decisión política para enfrentarla.

\section{Unidad de la naturaleza expresada en la ciencia}

El abordaje de la cuestión ambiental constituyó el gran desafío para las ciencias ecológicas y las ciencias sociales, que fuera advertido por Novik (1982) al afirmar que en el pluridemensional problema científico-socionatural de la optimización de la biosfera hay un núcleo metodológico relacionado con la tarea de lograr una integración eficaz de las ciencias.

La universalidad del problema ecológico exige un tratamiento integral; para ello fue imprescindible trabajar en la integración de las ciencias, pues la unidad de la naturaleza debe tener su expresión lógica en la unidad de ciencia. Este debió ser, por el imperativo de la supervivencia de la humanidad, el camino de la ciencia. La visión integradora de la ciencia fue ganando terreno en el mundo y a la vez reveló que revolución científico-tecnológica por sí sola es insuficiente para lograr un progreso universal (Novik, 1982).

En el estudio de la biosfera se advirtió la atención preferente al componente biótico, tal vez porque los seres vivientes representan la más elevada manifestación de la materia o quizá por cierta subvaloración del constituyente abiótico al que se le considera como permanente e inmutable. Sin embargo, los cambios ocasionados por la explotación extensiva de los recursos naturales, el crecimiento 
industrial, entre otras causas determinan graves y hasta peligrosas alternaciones de ciertos parámetros no biológicos como la reducción de la capa de ozono, el aumento de la temperatura de la atmósfera, la lluvia acida, etc., demandan incorporación de análisis físicos y químicos en los estudios de la biosfera.

Lo inerte y lo viviente constituyen una unidad. Lo viviente no solo se debe considerar como el producto de la evolución de ciertas estructuras químicas, sino el resultado de una forma de realidad física determinada por sus constantes. Lo viviente ha sido amoldado por la física existente de los procesos objetivos (Novik, 1982). Si lo viviente está determinado por el medio físico; el hombre no debe de ser ubicado fuera de la naturaleza. El hombre y su medio natural son subsistemas y forman parte de un sistema muy amplio y complejo que es el sistema geobiosocial; por consiguiente debe ser estudiado por las ciencias naturales y sociales, pues, el estado actual del sistema geobiosocial es en gran parte producto de la sociedad humana; aquí radica la razón de ser de la integración de las ciencias naturales, en especial de la ecología con la sociología y la economía. Pero tal síntesis será posible cuando se incluyan en las investigaciones de la naturaleza elementos axiológicos. La orientación axiológica de las ciencias naturales es una categoría metodológica. El monismo del materialismo dialéctico se opone al divorcio de la metodología y la axiología; por el contrario, su unidad, en las condiciones modernas, es una expresión importante de la unidad a la naturaleza y la sociedad, remarca Novik (1982). Empero, la unidad de las ciencias naturales y las sociales no ha de buscarse en el reduccionismo de las ciencias sociales a las primeras como pretende alguna escuela filosófica, sino a través de la integración recíproca que se logrará mediante el acercamiento de la verdad y el valor, es decir, la metodología y la axiología.

\section{Ciencia ecológica, pensamiento ambiental y humanismo}

El camino del nuevo quehacer científico con un nuevo enfoque, a la vez natural y social, abrió nuevos horizontes a la humanidad. De ese modo la hermosa aventura de la búsqueda de la verdad que es la ciencia asume una orientación humanista superior. Las mejores expresiones del espíritu humano como las ciencias, las artes, los valores éticos y por cierto la conciencia política entendida como preocupación y acción de solidaridad humana, alcanzan una unidad indisoluble. "Ciencia sin conciencia no es más que la ruina del alma”, advirtió Francois Rabelais, en los albores del Renacimiento.

La síntesis de las ciencias naturales y sociales abre nuevas perspectivas, que permiten conocer y administrar racionalmente la naturaleza en función del presente y del futuro del hombre. Vernadsky, fundador de la biogeoquímica y creador del concepto de biosfera advirtió del poder y la fuerza geológica de la humanidad, de la necesidad de conservar y restaurar la biosfera para bien de la humanidad ya que sin la biosfera no es posible la vida de Homo sapiens y sin él no es posible ningún progreso social, ninguna evolución social ni cultural.

La ecología emerge con gran fuerza en las últimas décadas, su vigor y presencia radican en la aptitud para abordar los problemas globales de la época, de la capacidad para hacer frente a las condiciones no previsibles del porvenir y a 
la posibilidad de señalar el camino para vivir en armonía con los demás y con la naturaleza. Según Víctor Manuel Toledo: La ciencia ecológica pone en evidencia que la naturaleza es el sustrato de toda producción primaria. Durante los procesos productivos los hombres agrupados en sociedades se apropian de ecosistemas más que de recursos naturales, es decir, de unidades-totalidades, dotadas de una estructura, función y un equilibrio determinado. De esta manera, la ecología ofrece un conjunto de conocimientos que hacen (o harán) posible la apropiación correcta de la naturaleza lo que implica su adecuada inserción en los procesos productivos. Este es, a criterio de muchos, el mayor aporte teórico y práctico de la ecología (Toledo, 1987).

La ecología es la ciencia de síntesis por excelencia y síntesis de la ciencia; ciencia de la naturaleza y del hombre que en años recientes ha contribuido a una transformación profunda en el ámbito de las ciencias sociales. La ecología moderna se ramifica y se interrelaciona con otras disciplinas, al punto que ha permitido un importante desarrollo del conocimiento: cabe mencionar algunas de las principales ramas e imbricaciones: ecología social, antropología ecológica, ecología política, economía ecológica, ecología de paisajes... La ecología moderna surgió como una exigencia de la aprehensión global del medio ambiente, también produjo una interesante cohesión con las ciencias humanas.

Nunca imaginaron los científicos naturales que pusieron en forma científica a las relaciones que se dan en los sistemas naturales, que no solo estaban investigando hechos naturales, sino además estaban abriendo una perspectiva política de dimensiones incalculables, es decir, una ciencia que iba a situarse en el centro de los debates políticos del siglo XX y XXI (Mires, 2003).

\section{Crisis ecológica, económica y civilizatoria}

La crisis ecológica y la crisis económica que sacude al mundo son la manifestación visible de graves desviaciones que parten de la antigua concepción del mundo occidental, que se resume en la dicotomía hombre versus naturaleza. Ese dualismo filosófico fundamentó el pensamiento económico de occidente y dio origen a la actual sociedad tecnológico industrial que transformó a la naturaleza en fuente gratuita de materia prima, en un bien de libre acceso de donde se podía explotar sin límites ni reservas, en una entidad amorfa susceptible de ser manipulada y transformada en objeto de intercambio; en suma, la naturaleza convertida en "capital natural" o lo que se ha dado en llamar la "capitalización de la naturaleza".

En la sociedad tecnológico industrial el aparato productivo funciona como un flujo abierto y unidireccional de materia y energía: por un lado ingresa materia prima y por el otro salen productos con diverso grado de transformación, además de desechos de todo tipo. Este sistema sigue operando sin límites en el tiempo y el espacio; sus expresiones concretas son una gigantesca producción industrial, la industria bélica incontenible, la producción para la sociedad de consumo cuya obsolescencia está programada de antemano, la carrera espacial con objetivos militares y de dominación geopolítica, las armas atómicas, la era de la computación, 
la informática y la robótica; todo lo cual opera por medio de complejos mega industriales altamente destructivos y contaminantes. El calentamiento global, la contaminación y degradación ambiental, la reducción de la diversidad biológica en escala planetaria son la consecuencia inevitable de ese modelo de sociedad de consumo y desperdicio.

La civilización industrial ignoró deliberadamente los principios de la ecología, olvidó que el ser humano es parte de la naturaleza y que se halla inmerso en su compleja dinamia. Esa omisión ha conducido a la crisis ecológica mundial que es solo una de las crisis de la modernidad, pues, la crisis económica y financiera que sacude al mundo es una crisis civilizatoria derivada de culto al mercado, al dinero y a la tecnología: una crisis de la civilización del egoísmo y de las cosas (Báez, 2000). Una crisis del sistema por la cual el planeta está en peligro, porque se sustenta en la injusticia social y en una profunda injusticia ambiental, que se generalizó "globalizando la explotación de la naturaleza", en expresión de Porto-Gonçalves (2006).

En este escenario de principios del siglo XXI las reflexiones académicas se orientan a examinar los principios y los conceptos de la economía, la sociología y la política y a buscar los vínculos entre las políticas económicas y las políticas ambientales con el propósito de lograr el desarrollo que garantice la perdurabilidad des los sistemas ecológicos mediante la consideración de las siguientes dimensiones: social fundamentada en la distribución equitativa de la riqueza, económica que se expresa en la eficaz repartición y gestión de los recursos, ecológica a través del respeto a la capacidad de autorreproducción de los ecosistemas, espacial en términos de mayor equilibrio campo-ciudad y de las actividades económicas del territorio, y, cultural a través de una pluralidad de soluciones locales que tomen en cuenta las diferencias culturales y la diversidad de los ecosistemas. (Da Ros, 1997).

En esta vertiente del pensamiento se sitúa la economía ecológica que considera viable la sustentabilidad en el marco de una relación adecuada entre los sistemas económicos y sistemas ecológicos; de equidad distributiva intra e intergeneracional, y de igualdad en las relaciones internacionales. La economía ecológica al estudiar la relación entre los sistemas económicos ofrece una explicación consistente de la mayor parte de los problemas actuales: calentamiento global, pérdida de biodiversidad, pobreza, distribución desigual de la riqueza y otros. La economía ecológica -ciencia y manejo de la sostenibilidad- requiere de un enfoque interdisciplinario que exige "orquestar las ciencias" y trabajar bajo un pluralismo metodológico (Falconí, 2002). Enfoque atractivo y promisorio pero aún insuficiente para enfrentar la compleja problemática socio ambiental que exige un análisis multidisciplinario desde la perspectiva antropológica, la filosófica, política y ética.

\section{Emergencia del nuevo pensamiento ambiental}

La vanguardia del pensamiento contemporáneo que emerge de la nueva síntesis de las ciencias y las humanidades está impulsando una nueva concepción del mundo que llevará a superar en forma definitiva la vieja concepción antroprocéntrica en la interpretación de la naturaleza, que es la responsable de la crisis ambiental y económica global que vive la humanidad. Después del fracaso de 
viejos paradigmas, la nueva síntesis del conocimiento científico y social hallará nuevos caminos para la humanidad en el "nuevo paradigma ambiental" que se construye en el marco del pensamiento ambiental emergente en los albores del nuevo milenio con la confluencia de lo más lúcido del conocimiento generado por las ciencias naturales y las ciencias sociales. Cabe destacar que las ciencias sociales se han enriquecido en forma significativa con el aporte de la teoría ecológica y se espera que la sociología del medio ambiente, así como la economía política del medio ambiente, revitalicen y orienten el pensamiento social y político.

La crisis ambiental, económica y social de la modernidad que ha llegado a su clímax en las primeras décadas del siglo XXI exige el abordaje de esa problemática desde una perspectiva más amplia e integral en el contexto de la filosofía ambiental. En efecto, el pensamiento ambiental y la construcción de una filosofía que incorpore la dimensión ambiental están ganado terreno en la reflexión académica contemporánea, como una respuesta al excesivo reduccionismo del pensamiento hegemónico. Este pensamiento nacido de la cultura dominante ha ejercido el "efecto sombra" al negar la diversidad de perspectivas de las sociedades en cuanto a sus valores, sistemas de economía y valoración ética. El "efecto sombra" de los modelos políticos y económicos dominantes debe ser superado; es un imperativo para la supervivencia de la humanidad "abrir claros" que permita expresar la mega diversidad de valores éticos y anhelos de la vida latentes en América Latina (Primack, et al., 2001).

\section{Construcción del pensamiento ambiental}

El nuevo pensamiento ambiental busca integrar lo más avanzado del pensamiento científico y social contemporáneo a la vez que recuperar cosmovisiones, principios y valores que conservan los pueblos y culturas milenarias de oriente y occidente. Está constituyéndose en una construcción teórica ínter y transdisciplinaria que cruza de manera transversal la totalidad de las disciplinas; que permite construir conceptos, valores, símbolos y prácticas transformadoras de la sociedad, que lleve a las personas y a la humanidad a actuar con responsabilidad ambiental, para que el Homo sapiens se asuma como naturaleza y actúe bajo una nueva ética. Esto exige una reingeniería del pensamiento mediante aproximaciones interdisciplinarias como la ética ambiental, el derecho ecológico, la economía ecológica, la antropología ecológica, la ecología política, la historia ambiental, el periodismo ecológico y la ecología humana. La aproximación transdisciplinaria no supone abolir las disciplinas sino su integración dentro de un enfoque holístico y sistémico en el marco de la filosofía ambiental. Es fundamental salvar las distancias entre las humanidades y las ciencias, remarca Primack, et al. (2001).

Antes de avanzar en el análisis de la construcción del pensamiento ambiental es necesario precisar la naturaleza, el ámbito, el alcance de lo ambiental. Para su definición se debe partir del estudio de la ecología que permite la aprehensión de los organismos y su relación con el entorno natural y el funcionamiento de los sistemas ecológicos; pero además es preciso considerar los diferentes sistemas culturales que surgen en la evolución de las formaciones sociales a través de la historia de la humanidad. 
La visión de lo ambiental desde la perspectiva ecológica es básica y fundamental pero no es suficiente para entender lo ambiental en toda su complejidad. El enfoque ecológico es solo la primera parte del análisis ambiental. (Esta es la comprensión que predomina en los movimientos ecologistas, por lo que su discurso se limita a una retórica de buenas intenciones y a una práctica conservacionista y de acciones puntuales, sin considerar de manera suficiente los factores sociales y culturales de los países y regiones donde actúan). Es necesario, por lo mismo, distinguir lo que se denomina ambiente en ecología y lo que comprende el ambiente en los sistemas socioculturales, en los ecosistemas no intervenidos y los ecosistemas trasformados por intervención humana.

Es preciso diferenciar las transformaciones ecosistémicas naturales y las transformaciones tecnológicas inducidas por los sistemas culturales ya que éstos modifican sustancialmente el sistema ecológico. A medida que la naturaleza es intervenida tiene cada vez menos posibilidad de regresar a las condiciones primitivas; por lo mismo, se busca encontrar nuevos equilibrios, esos nuevos equilibrios sustentan y sustentarán a las sociedades. Esos equilibrios que son llamados "tecnobiológicos" sintetizan las transformaciones tecnológicas sobre la base de los ecosistemas y los recursos naturales; por lo mismo, es necesario comprender las complejas articulaciones del sistema social para entender la naturaleza en su conjunto. Esto significa que el orden natural incluye en la actualidad el orden humano. Empero, el orden humano no coincide con el orden ecosistémico, por lo que la especie humana no tiene ninguna alternativa evolutiva sino la transformación el orden ecosistémico. La solución a la problemática ambiental no consiste en "conservar" sino en "transformar bien". Tal transformación incluye herramientas, equipos y todo tipo de medios físicos, pero también las formas de organización social, económica y cultural, así como una red de símbolos que cohesionan los sistemas sociales, según el esclarecedor análisis de Augusto Ángel. (Ángel, 1995)

La discusión de lo ambiental es un fenómeno reciente; nace de la necesidad de diferenciar las entidades: ecosistema y ambiente. El ambiente no se circunscribe a la ecología -enfatiza Augusto Ángel-. El ambiente es mucho más amplio y complejo porque incluye las relaciones entre la naturaleza y la cultura, lo material y lo simbólico, el ser individual y el ser social. El ambiente es una entidad altamente compleja, heterogénea y cambiante en el tiempo y el espacio. Está conformada por una conjunción compleja de entidades naturales y socio-culturales. El ambiente debe ser entendido como entidad natural, pero en el marco de las especificidades culturales. Las responsabilidades ambientales del hombre no podrán ser entendidas, mientras no se entienda la cultura como una emergencia evolutiva que tiene sus propias reglas de funcionamiento, como las tienen los ecosistemas (Ángel, 1995).

\section{Complejidad del pensamiento ambiental}

La estructuración del pensamiento ambiental está todavía en proceso. En expresión de Augusto Angel:

La ciencia moderna, dominada por el positivismo, ha impulsado la especialización como estrategia básica del desarrollo del conocimiento lo que exige la especiali- 
zación que parte tanto de presupuestos del racionalismo cartesiano como de los postulados del empirismo. La consecuencia ha sido la incapacidad de la ciencia moderna para entender y manejar sistemas y, por supuesto, para ubicar al hombre dentro del sistema de la naturaleza. Otro factor que incide fuertemente en la estructuración del pensamiento ambiental son las distintas perspectivas ambientales que han surgido en las últimas décadas y que representan diferentes acercamientos disciplinarios surgidos en el ámbito de la ciencia contemporánea como la ecología, la economía, las ciencias sociales, la antropología, la psicología ambiental, la historia ambiental, la ingeniería... en suma un conjunto de aproximaciones en lo que se ha denominado pomposamente "ciencias ambientales" que buscan comprender uno de los problemas del hombre: su articulación exitosa o catastrófica, pero siempre problemática, con el medio ecosistémico (Angel, 1995).

En el abordaje del problema ambiental se ha producido una fructífera confluencia de las ciencias naturales y ciencias humanas. El pensamiento ambiental logra integrar una perspectiva humanística centrada en la discusión ambiental que ha sido asumida por las ciencias físicas, aborda lo epistémico, sociopolítico, lo ético, lo cultural y pedagógico. El pensamiento ambiental es un pensar que es fruto de la acción y experiencia humana de los últimos años, de ahí que sea un cuerpo teórico en construcción, abierto a la utopía, a la vez que crítico de las experiencias sociales, según Cajigas-Rotundo (2008).

\section{El saber y la racionalidad ambiental}

El saber ambiental es un saber en proceso de construcción que emerge de la reflexión sobre la naturaleza. En su esclarecedor análisis Ernesto Leff sostiene que el saber ambiental:

Es el campo donde convergen diversas epistemologías, racionalidades e imaginarios que transforman la naturaleza, construyen la realidad, va entretejiendo una trama compleja de conocimientos y cosmovisiones, que abre un diálogo de saberes en donde se confrontan diversas racionalidades y tradiciones. Es un saber que emerge desde el límite del pensamiento unidimensional de la razón objetivadora y cosificadora, y se construye con el encuentro de identidades y saberes. Pero además el saber ambiental revela las estrategias de poder, a la vez que establece las bases para pensar y construir una nueva racionalidad para la comprensión y construcción de un mundo sustentable. El saber ambiental produce un cambio de episteme al abordar la relación entre el ser y el saber. Da curso a un nuevo saber que emerge desde la marca de un límite, de una ley-límite de la naturaleza, de la ineluctable ley de la entropía. La epistemología ambiental reconoce los efectos de las formas de conocimiento en la construcción/destrucción de la realidad; aporta a la comprensión, significación y apropiación del mundo (Leff, 2004).

El discurso del desarrollo sustentable que tuviera tanta difusión en las últimas décadas -particularmente desde la ONU, las agencias internacionales de desarrollo, ONGs y entidades nacionales de planificación- fue severamente cuestionado por ser vacío de contenido real, abstracto, tautológico y por representar un planteamiento del poder hegemónico que busca perennizar el statu quo; llevó también 
a repensar el concepto de lo ambiental, la complejidad ambiental y la racionalidad ambiental. En expresión de Leff:

\begin{abstract}
Aquí se plasma la propuesta teórico-filosófico-político de construcción de una racionalidad ambiental en un campo práctico, donde el potencial ecológico, la productividad tecnológica y la creatividad cultural se amalgaman en nuevas estrategias agropecuarias y agroforestales, en un diálogo de saberes entre las ciencias ecológicas y agronómicas con los saberes indígenas y campesinos, en un proceso de reapropiación cultural, técnica y social de la naturaleza (Leff, 2009).
\end{abstract}

\title{
La voz de la iglesia
}

La encíclica "Laudato si' sobre el cuidado de la casa común" del papa Francisco publicada el 18 de junio de 2015 es el resultado de profundas reflexiones compartidas con científicos de la Academia de Ciencias del Vaticano, filósofos, teólogos, y organizaciones sociales que han enriquecido el pensamiento de la Iglesia Católica sobre cuestiones fundamentales de la naturaleza y el medio ambiente. Laudato si' ("Alabado seas", en español), parte de la convicción de que en el mundo todo está conectado, establece la relación entre pobreza y la fragilidad del planeta; critica a las formas de poder ligado a la tecnología; invita a buscar otros modos de entender la economía y el progreso; destaca el valor de cada criatura, el sentido humano de la ecología y aboga por un nuevo estilo de vida.

En la encíclica el papa Francisco cuestiona el paradigma tecnocrático por ser homogéneo y unidimensional, el antropocentrismo desviado que ha dado lugar a un estilo de vida desviado, sostiene que la crisis ecológica es una manifestación externa de la crisis ética, cultural y espiritual de la modernidad, propone avanzar hacia una ecología integral que incorpore las dimensiones humanas y sociales; el principio de bien común, la justicia generacional a la vez que aboga por la conversión ecológica. Con la carta encíclica el Papa latinoamericano - principal líder espiritual mundial- llama a la reflexión sobre "los 'mitos' de la modernidad basados en la razón instrumental (progreso indefinido, competencia, consumismo, mercado sin reglas) y exhorta a recuperar los distintos niveles de equilibrio ecológico (...)". Así, aporta a la discusión de la problemática ambiental y planetaria con lo cual contribuye a enriquecer el pensamiento ambiental partiendo de la ciencia, la teología, la axiología... (Francisco, 2015).

"La encíclica ecológica -como se le ha denominado- sitúa a la Iglesia en la vanguardia de las propuestas emancipadoras, muy por delante del ambientalismo y ecologismo clásicos, el marxismo, el nuevo socialismo (...) el indigenismo radical (...).” Además,

Es un manifiesto revolucionario que da un giro a los fundamentos teológicos de la Iglesia, recoge las tesis más avanzadas del pensamiento crítico y del pensamiento complejo, identifica a los agentes de la depredación y explotación y ofrece una plataforma ideológica para la acción de los sectores excluidos (Toledo, 2015).

En la encíclica Laudato si' el Papa llama a salvar el planeta y a buscar "caminos de vida nueva". 
Desde diversas conceptualizaciones teóricas se aborda la problemática ambiental del mundo actual. Patricia Noriega de Echanique afirma:

El pensamiento ambiental es esa obligatoria reflexión que debe realizar hoy todo aquel que se pregunta el por qué y el para qué conocemos. Es ese imperativo moral surgido del abismo ante el cual estamos hoy como partícipes de este planeta, como hilos de la trama de la vida.

Contrasta dos sistemas de pensamiento:

El pensamiento ambiental se despliega en la integralidad de los modos de ser del ser, mientras que el pensamiento moderno aparece como el dominio de unos modos de ser sobre otros. El pensamiento ambiental invita a la construcción de saberes solidarios (...) Realiza cruces, transversaliza ideas (...) (Noguera, 2004).

\section{El pensamiento ambiental latinoamericano}

En años recientes intelectuales y académicos de América Latina buscan estructurar un pensamiento ambiental genuinamente latinoamericano como respuesta al discurso del desarrollo sustentable, revaloran el concepto de sustentabilidad y destacan las relaciones entre el saber y el hacer de los pueblos de nuestra Patria Grande.

El pensamiento ambiental latinoamericano que se va configurando es un repensar el mundo desde las raíces ecológicas y culturales de nuestros territorios (...). "Se ha venido configurando un pensamiento ambiental latinoamericano, un pensamiento con identidad propia que está arraigado en los territorios de vida de sus pueblos y naciones, en nuestros sistemas educativos, que está fertilizando modos de producción y nuevas formas de convivencia basados en la cultura de los potenciales ecológicos de la región y en una ética del cuidado de la vida. (Leff, 2009).

El pensamiento y el saber ambiental tienen un potencial de transformación social muy grande que apenas se empieza a vislumbrar. La epistemología ambiental que emerge con inusitada fuerza en este siglo se espera que contribuya a la consolidación de un nuevo pensar y un nuevo ser.

La racionalidad ambiental se forja en la deconstrucción del pensamiento metafísico, científico y posmoderno, de la territorialización de la diversidad y la otredad; sobre la base de los potenciales ecológicos y de los saberes culturales que habitan en los territorios del Sur. De allí nace y desde allí reivindicamos el pensamiento ambiental latinoamericano (Leff 2009).

El nuevo pensamiento, saber y racionalidad ambiental resultante de la síntesis de las ciencias naturales, las ciencias sociales y las ciencias humanas está ganando un importante espacio en las reflexiones académicas de la presente década y se espera que ilumine el camino de la humanidad en este siglo, que es el siglo del medio ambiente. 


\section{Bibliografía}

Ángel, A. (1995). Desarrollo sostenible: aproximaciones conceptuales. Quito: Publicación de Fundación NATURA, Fundación Ecuatoriana para la Conservación de la Naturaleza, UIC

Báez, R. (2000). El doble derrumbe de la modernidad: un enfoque ético.

En www.alainet/org/active/. 15/12/2008.

Cajigas-Rotundo, J. (2008). Pensamiento ambiental: un pensar perfectible. Revista Ideas Ambientales. En www.esayistas.org/critica/ecologia/cajigas. 08/08/2015.

Da Rose, G. (1997). Economía ambiental y gestión ecológica de la empresa. En Laboratorio de Economía. Revista de la Facultad de Economía de la Universidad Católica del Ecuador, año I, nro.1. Quito, pp. 21-28.

Di Castri, F. (1981). La ecología moderna: génesis de una ciencia del hombre y de la naturaleza. En El Correo de la Unesco, pp. 6-11.

Falconí, F. (2002). Economía y desarrollo sostenible. ¿Matrimonio feliz o divorcio anunciado? El caso de Ecuador. FLACSO, Sede Quito.

Francisco (2015). Encíclica del papa Francisco: Laudato si' sobre el cuidado de la casa común. http://www.acipresa.com/Docum/Laudato $\mathrm{Si}^{\prime}$ pdf 01/07/2015.

Gimaráes, R. (1992). El discreto encanto de la Cumbre de la Tierra. Evaluación Impresionista de Río-92. En Rev. Nueva Sociedad, nro. 122, Caracas, Venezuela, pp. 86-106.

Hutchinson, G. E. (1972). La biosfera. En La biosfera, Scientific American. Madrid: Alianza Editorial.

Kormondy, E. (1973). Conceptos de ecología. Madrid: Editorial Alianza.

Leff-Zimmerman, E. (2004). Educación ambiental: perspectivas desde el conocimiento, la ciencia, la ética, la cultura, la sociedad y la sustentabilidad. En Revista de Ideas Ambientales, ed. nro. 1, Manizales, Colombia.

Leff, E. (2009). Pensamiento ambiental latinoamericano: patrimonio de un saber para la sustentabilidad. www.cep.unt.edu./paper/leff-span-pdf/13/07/2015.

Margalef, R. (1980). Ecología. Barcelona, España: Editorial Omega.

Margalef, R. (1983). Ecología (3ra. ed.). Barcelona, España: Editorial Planeta.

Mires, F. (2003). Lo ecológico y lo político. Política ecológica y derechos humanos. En El Oriente es un mito. Quito: Ediciones Abya-Yala, pp. 190-221.

Noguera, P. (2004). El reencantamiento del mundo. http://www.unter.org.ar/imagenes/ PDF 06/08/2015.

Novik, I. (1982). Sociedad y naturaleza. Moscú: Editorial Progreso.

Odum, E. (1969). Ecología (2da. ed.). México: Editorial Interamericana. 
Porto-Gonçalves, C. (2006). Del efecto invernadero y de los efectos del capitalismo. En Revista América Latina en Movimiento, nro. 412, 16-21.

Primack, R., Rozzi, R., Feisinger, P., Dirzo, R., Massardo, F. (2001). Fundamentos de conservación biológica. Perspectivas latinoamericanas. México: Editorial Fondo de Cultura Económica.

Sarmiento, F. (2001). Diccionario de ecología: paisajes, conservación y desarrollo sustentable para América Latina. Quito: Abya-Yala, Cepeige.

Toledo, V. M. et al. (1987). Ecología y autosuficiencia alimentaria (2da. ed.) México: Siglo XXI Editores.

Toledo, V.M. (2015). La ecología subversiva del papa Francisco. En La Jornada, México. www.jornada.unam.mx/2015/06/23.opinion/018a1 pol. $07 / 08 / 2015$. 